\title{
Strong-Disorder Paramagnetic-Ferromagnetic Fixed Point in the Square-Lattice $\pm J$ Ising Model
}

\author{
Francesco Parisen Toldin • Andrea Pelissetto • \\ Ettore Vicari
}

Received: 13 November 2008 / Accepted: 25 February 2009 / Published online: 14 March 2009

(C) Springer Science+Business Media, LLC 2009

\begin{abstract}
We consider the random-bond $\pm J$ Ising model on a square lattice as a function of the temperature $T$ and of the disorder parameter $p$ ( $p=1$ corresponds to the pure Ising model). We investigate the critical behavior along the paramagnetic-ferromagnetic transition line at low temperatures, below the temperature of the multicritical Nishimori point at $T^{*}=$ $0.9527(1), p^{*}=0.89083(3)$. We present finite-size scaling analyses of Monte Carlo results at two temperature values, $T \approx 0.645$ and $T=0.5$. The results show that the paramagneticferromagnetic transition line is reentrant for $T<T^{*}$, that the transitions are continuous and controlled by a strong-disorder fixed point with critical exponents $v=1.50(4), \eta=$ $0.128(8)$, and $\beta=0.095(5)$. This fixed point is definitely different from the Ising fixed point controlling the paramagnetic-ferromagnetic transitions for $T>T^{*}$. Our results for the critical exponents are consistent with the hyperscaling relation $2 \beta / \nu-\eta=d-2=0$.
\end{abstract}

Keywords Two-dimensional Edwards-Anderson model · Two-dimensional Ising spin glass · Critical exponents $\cdot$ Monte Carlo simulations $\cdot$ Strong-disorder fixed point

F. Parisen Toldin

Max-Planck-Institut für Metallforschung, Heisenbergstrasse 3, 70569 Stuttgart, Germany

F. Parisen Toldin

Institut für Theoretische und Angewandte Physik, Universität Stuttgart, Pfaffenwaldring 57,

70569 Stuttgart, Germany

A. Pelissetto $(\bowtie)$

Dipartimento di Fisica, Università di Roma "La Sapienza” and INFN, Piazzale Aldo Moro 2,

00185 Roma, Italy

e-mail: Andrea.Pelissetto@roma1.infn.it

E. Vicari

Dipartimento di Fisica, Università di Pisa and INFN, Largo Pontecorvo 3, 56127 Pisa, Italy 


\section{Introduction}

The $\pm J$ Ising model represents an interesting theoretical laboratory, in which one can study the effects of quenched disorder and frustration on the critical behavior of spin systems. While originally introduced to describe magnetic systems with disordered couplings [1], it has been shown recently to be also relevant for quantum computations [2, 3]. It is defined by the lattice Hamiltonian [1]

$$
\mathcal{H}=-\sum_{\langle x y\rangle} J_{x y} \sigma_{x} \sigma_{y}
$$

where $\sigma_{x}= \pm 1$, the sum is over all pairs of lattice nearest-neighbor sites, and the exchange interactions $J_{x y}$ are uncorrelated quenched random variables, taking values $\pm J$ with probability distribution

$$
P\left(J_{x y}\right)=p \delta\left(J_{x y}-J\right)+(1-p) \delta\left(J_{x y}+J\right) .
$$

In the following we set $J=1$ without loss of generality. For $p=1$ we recover the standard Ising model, while for $p=1 / 2$ we obtain the bimodal Ising spin-glass model.

The $T$ - $p$ phase diagram of the two-dimensional (2D) square-lattice $\pm J$ Ising model has been extensively investigated [4-46]. The resulting phase diagram, which is sketched in Fig. 1, presents two phases at finite temperature: a paramagnetic and a ferromagnetic phase. They are separated by a transition line, which starts at the pure Ising transition point at $p=1$ and $T_{\mathrm{Is}} \approx 2.269$ and ends at the $T=0$ transition at $p_{0} \approx 0.897$. The point where this transition line meets the so-called Nishimori (N) line [13], at $T^{*}=0.9527(1)$ and $p^{*}=$ 0.89083 (3) (we derive these estimates in the present paper), is a multicritical point (MNP) [36, 37].

The MNP divides the paramagnetic-ferromagnetic (PF) transition line in two parts. The PF transition line from the Ising point at $p=1$ to the MNP is controlled by the Ising fixed point: disorder gives only rise to logarithmic corrections to the standard Ising critical behavior [11]. On the other hand, the presence of the MNP on the transition line suggests that the PF transitions for $T<T^{*}$ belong to a different strong-disorder universality class. This is

Fig. 1 (Color online) Phase diagram of the square-lattice $\pm J$ Ising model for $1-p \leq 1 / 2$. SDI labels the critical transition line associated with the strong-disorder fixed point

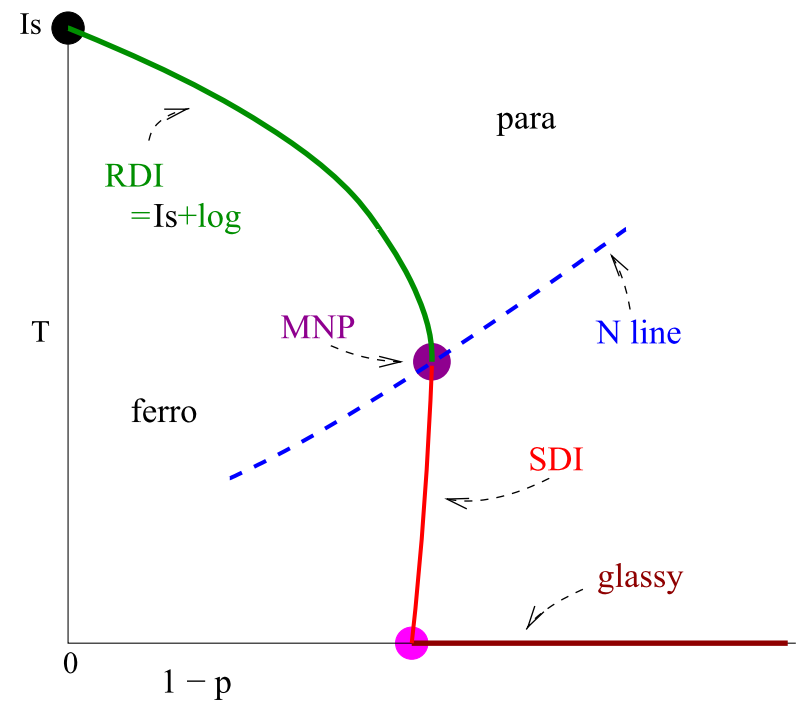


confirmed by the renormalization-group (RG) calculations of [43] and [30], using domainwall and Migdal-Kadanoff RG transformations, respectively, which found that the RG flow along the critical line for $T<T^{*}$ was attracted by a different fixed point.

In this paper we investigate the critical behavior along the low-temperature transition line from the MNP to the $T=0$ axis. We perform Monte Carlo (MC) simulations at two temperature values below the MNP, i.e., at $\beta \equiv 1 / T=2$ and $\beta=1.55$. As we shall see, our finite-size scaling (FSS) analyses show that the PF transition line for $T<T^{*}$ is reentrant and that the transitions are continuous. Moreover, the estimates of the critical exponents and of several RG invariant quantities for these two values of $T$ are consistent, supporting the hypothesis that the PF transition line below the MNP belongs to a unique universality class. The values of the critical exponents, $v=1.50(4), \eta=0.128(8)$, and $\beta=0.095(5)$ are clearly different from the Ising values $v=1, \eta=1 / 4, \beta=1 / 8$. Therefore, these results show the existence of a strong-disorder fixed point associated with a PF transition. Note that this strong-disorder fixed point does not violate hyperscaling. Indeed, our results are consistent with the hyperscaling relation $2+2 \beta / v-\eta=d=2$ (our estimates of the critical exponents $\eta$ and $\beta$ give $2+2 \beta / v-\eta=2.00(1)$ ). The transitions for $T<T^{*}$ are no longer in the basin of attraction of the Ising fixed point, which is the relevant one for small disorder and determines the critical behavior along the transition line for $T>T^{*}$.

The paper is organized as follows. In Sect. 2 we review the main features of the $T-p$ phase diagram of the square-lattice $\pm J$ Ising model. The MC results and their FSS analyses are presented in Sect. 3. In Sect. 4 we draw our conclusions. Some technical details on the simulations are presented in Appendix A, while the quantities we compute are defined in Appendix B. In Appendix C we present a reanalysis of the critical behavior at the MNP, using the additional data we have collected in this work. Moreover, we also present analyses which take into account the analytic corrections, which had been neglected in our previous work [15]. This allows us to obtain improved estimates of the critical parameters at the MNP.

\section{The Phase Diagram of the Square-Lattice $\pm J$ Ising Model}

The phase diagram of the square-lattice $\pm J$ Ising model is sketched in Fig. 1. It is symmetric for $p \rightarrow 1-p$ and thus we only report it for $1-p \leq 1 / 2$. For sufficiently small values of the probability of antiferromagnetic bonds $p_{a} \equiv 1-p$, the model presents a paramagnetic phase and a ferromagnetic phase, separated by a transition line. The PF transition line starts at the Ising point $X_{\mathrm{Is}}=\left(T=T_{\mathrm{Is}}, p=1\right)$, where $T_{\mathrm{Is}}=2 / \ln (1+\sqrt{2})=2.26919 \ldots$ is the critical temperature of the $2 \mathrm{D}$ Ising model, and extends up to a $T=0$ transition at $[44,45]$ $X_{0}=\left(T=0, p=p_{0} \approx 0.897\right)$.

The slope of the transition line at $p=1$ is exactly known [47]; for small $1-p$ we have

$$
T_{c}(p)=T_{\mathrm{Is}}\left[1-\frac{2 \sqrt{2}}{\ln (1+\sqrt{2})}(1-p)+\cdots\right] .
$$

In the $T-p$ phase diagram an important role is played by the Nishimori $(\mathrm{N})$ line $[4,13]$ defined by the equation $(p \geq 1 / 2)$

$$
T=T_{\mathrm{N}}(p), \quad T_{\mathrm{N}}(p)=\frac{2}{\ln p-\ln (1-p)} .
$$


Along the N-line several rigorous results can be proved [4, 13, 48]. The energy density is given by

$$
E_{\mathrm{N}}(p) \equiv \frac{1}{V}\left[\langle\mathcal{H}\rangle_{T_{\mathrm{N}}(p)}\right]=2-4 p,
$$

and the spin-spin and the overlap correlation functions are equal

$$
\left[\left\langle\sigma_{0} \sigma_{x}\right\rangle\right]=\left[\left\langle\sigma_{0} \sigma_{x}\right\rangle^{2}\right] .
$$

Here the angular and square brackets refer to the thermal average and to the quenched average over the bond couplings $\left\{J_{x y}\right\}$, respectively. As argued in [36, 37, 40, 41] and verified numerically $[15,18-20,22,33]$, the critical point $X_{\mathrm{MNP}}=\left(T^{*} \approx 0.953, p^{*} \approx 0.891\right)$ along the $\mathrm{N}$ line is a multicritical point (MNP).

Along the transition line from the Ising point $X_{\mathrm{Is}}$ to the MNP, the critical behavior is analogous to that observed in 2D randomly dilute Ising (RDI) models [11]. It is controlled by the pure Ising fixed point and disorder is marginally irrelevant, giving rise to a universal pattern of logarithmic corrections, see, e.g., [11, 49-54] and references therein.

The location of the MNP and the corresponding critical exponents can be obtained by FSS analyses of MC data along the $\mathrm{N}$ line. The new analysis reported in Appendix $\mathrm{C}$ gives

$$
T^{*}=0.9527(1), \quad p^{*}=0.89083(3) \text {. }
$$

In the absence of external fields, the MNP is characterized by two relevant RG operators with RG dimensions $y_{1}=0.66(1)$ and $y_{2}=0.250(2)$. Moreover, the magnetic exponent $\eta$ is given by $\eta=0.177(2)$. Other estimates of $T^{*}, p^{*}$, and of the critical exponents can be found in [14-16, 18, 22].

As a consequence of the inequality [13]

$$
\left|\left[\left\langle\sigma_{x} \sigma_{y}\right\rangle_{T}\right]_{p}\right| \leq\left[\left|\left\langle\sigma_{x} \sigma_{y}\right\rangle_{T_{N}(p)}\right|\right]_{p}
$$

(the subscripts indicate the values of $T$ and $p$ at which the thermal and disorder average are performed), ferromagnetism can only exist in the region $p \geq p^{*}$. Thus, the PF boundary lies in the region $p \geq p^{*}$ and, at the MNP, the transition line is tangent to the line $p=p^{*}$, hence parallel to the $T$ axis. As a further consequence, at $T=0$ the ferromagnetic phase ends at $p=p_{0}$ with $p_{0} \geq p^{*}$. In $[4,34,35,39]$ it was argued that the PF transition line from the MNP to $X_{0}=\left(0, p_{0}\right)$ is only related to the frustration distribution; hence, it should not depend on temperature and should coincide with the line $p=p^{*}$, so that $p_{0}=p^{*}$. Numerical estimates of $p_{0}$ have shown that this argument is not exact. Indeed, numerical analyses $[12,18,22,44-46]$ give $p_{0} \approx 0.897 ;^{1}$ this suggests that the transition line below the MNP is reentrant, i.e. $p_{c}>p^{*}$ for any $T<T^{*}$. The difference is however quite small, $p_{0}-p^{*} \approx 0.006$.

Our FSS analyses confirm that the PF transition line is reentrant for $T<T^{*}$. Indeed, we find $p_{c}=0.8915(2)$ at $T=1 / 1.55 \approx 0.645$ and $p_{c}=0.8925(1)$ at $T=0.5$. The PF transitions are of second order and show the same critical behavior with critical exponents $v=1.50(4), \eta=0.128(8)$, and $\beta=0.095(5)$, which are consistent with hyperscaling. These results confirm the existence of a strong-disorder fixed point, different from the Ising fixed point which controls the PF transitions above the MNP, i.e. for $T^{*}<T<T_{\text {Is }}$.

\footnotetext{
${ }^{1}$ The most precise estimates are apparently [44] $p_{0}=0.897(1)$ and [45] $p_{0}=0.8969(1)$.
} 
At variance with the three-dimensional case, there is no evidence of a finite-temperature glassy phase. Glassy behavior is only expected for $T=0$ and $p<p_{0}$. The critical behavior for $T \rightarrow 0$ has been much investigated for $p=1 / 2$ [6-10]. In particular, simulations found that the correlation length increases as $T^{-v}$ with $v \approx 3.5$. A natural hypothesis is that a $T=0$ glassy transition occurs for any $p<p_{0}$, with a critical behavior in the same universality class as that of the bimodal model with $p=1 / 2$.

The point $X_{0}=\left(0, p_{0}\right)$, where the low-temperature transition line ends is a multicritical point: it is connected to three phases and it is the intersection of two different transition lines, the PF line at $T>0$ and the glassy line at $T=0$. At $T=0$ the critical point $X_{0}$ separates a ferromagnetic phase from a $T=0$ glassy phase, while for $T>0$ the transition line separates a ferromagnetic from a paramagnetic phase. Therefore, on general grounds, the critical behavior when varying $p$ at $T=0$ differs from that along the PF transition line at $T>0$, unless the magnetic and glassy critical modes are effectively decoupled at the $T=0$ multicritical point. The latter scenario is apparently supported by the fact that the estimates of magnetic critical exponents at $T=0$ (see, e.g., [18, 43-45]) are quite close and substantially consistent with those found along the PF transition line for $0<T<T^{*}$.

\section{Monte Carlo Results}

We investigate the critical behavior along the PF line that starts at the MNP and ends at $T=0$. Since the transition line below the MNP is expected to be almost parallel to the $T$ axis, we study the FSS behavior of several quantities at fixed $T$ as a function of $p$. We consider two values of $T, \beta \equiv 1 / T=2$ and $\beta=1.55$, which are quite far from the two endpoints of the line. For each of these two values we perform MC simulations on square lattices of linear size $L$ with periodic boundary conditions, for several values of $L$ : $L=8,12,16,24,32,48,64$. In our MC simulations we employ the Metropolis algorithm, the random-exchange method (often called parallel-tempering or multiple Markov-chain method) [55-57], and multispin coding. Some details are reported in Appendix A.

\subsection{The Critical Point $p_{c}$ and Exponent $v$}

We first focus on the data at $\beta=2$, for which we have most of the statistics. In order to estimate $p_{c}$ and $v$, we perform a FSS analysis of the renormalized couplings $R_{\xi}, U_{4}, U_{22}$, which are defined in Appendix B and are generically denoted by $R$ in the following. MC estimates are shown in Fig. 2 . We clearly observe a crossing point for $0.8920<p<0.8930$, indicating $p_{c} \approx 0.892-0.893$, which is larger than the value at the MNP, i.e. $p_{c}=p^{*}=$ $0.89083(3)$. This already suggests that the transition line is reentrant.

To obtain more precise estimates we perform a careful FSS analysis, following [58]. In the FSS limit any RG invariant quantity obeys the scaling law

$$
R=f_{R}\left(u_{1} L^{y_{1}}\right),
$$

where $f_{R}(0)=R^{*}, y_{1} \equiv 1 / v$, and we have neglected scaling corrections. Here $u_{1}$ is the nonlinear scaling field associated with the leading relevant operator, which has RG dimension $y_{1}$. The scaling field is an analytic function of the system parameters which vanishes along the critical line. Thus, for $p \rightarrow p_{c}(\beta)$ at fixed $\beta$ we can write

$$
u_{1}=A_{0}(\beta)\left(p-p_{c}\right)+A_{1}(\beta)\left(p-p_{c}\right)^{2}+\cdots
$$


Fig. 2 (Color online) MC estimates of $R_{\xi} \equiv \xi / L, U_{4}, U_{22}$ at $\beta=2$ vs. $p$. The lines connecting the data at given $L$ are drawn to guide the eye. The dashed vertical lines correspond to the MNP location $p^{*}=0.89083(3)$. The dotted vertical lines indicate our final estimate of $p_{c}, p_{c}=0.8925(1)$
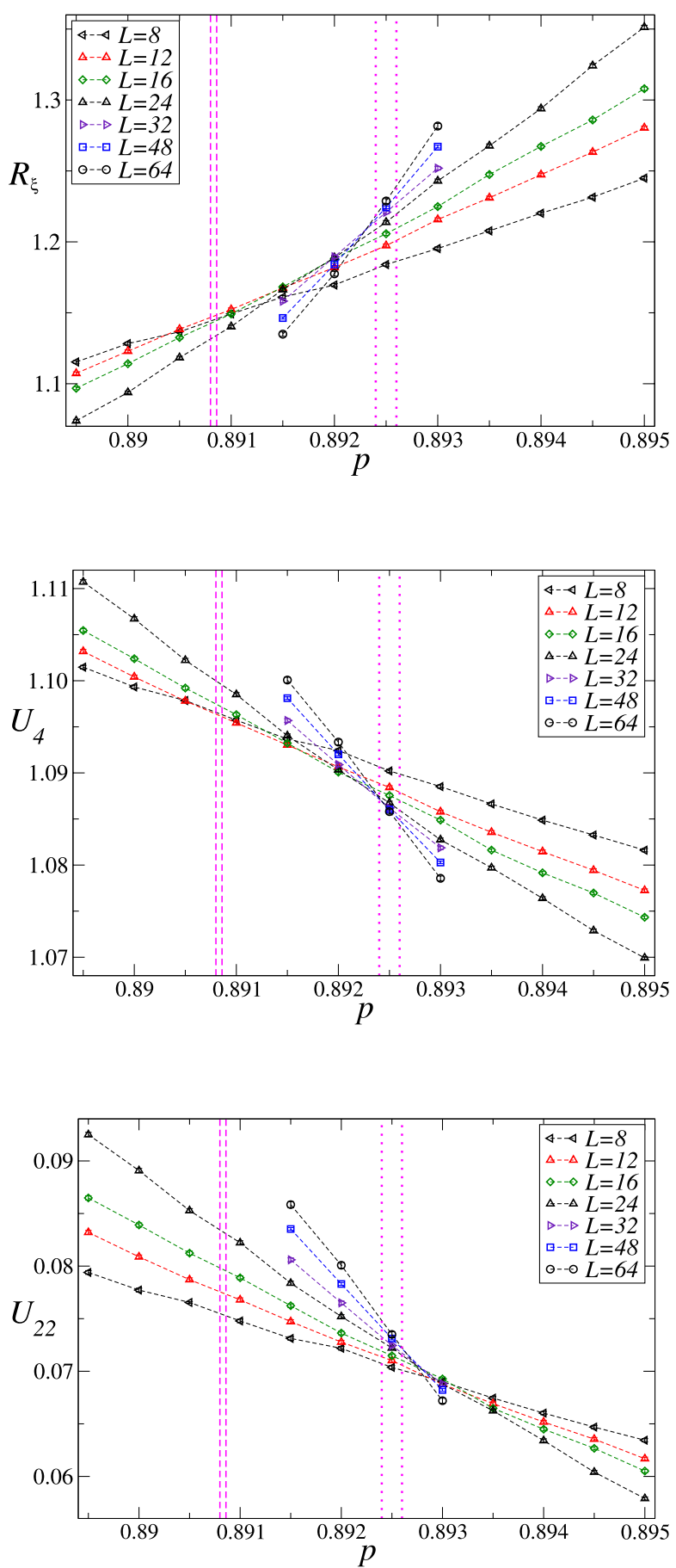
where the coefficients $A_{i}(\beta)$ are analytic functions of $\beta$. The terms of order $\left(p-p_{c}\right)^{2}$, $\left(p-p_{c}\right)^{3}$, etc., give rise to corrections of order $L^{-n y_{1}}$ as $L \rightarrow \infty$. They are named analytic corrections, because they arise from the analytic dependence of the scaling fields on the model parameters. See [58] for a thorough discussion of their origin. In pure ferromagnetic systems, in which $v \lesssim 1$ and $y_{1} \gtrsim 1$, they are usually negligible, and the nonanalytic corrections, which behave as $L^{-\omega}, \omega \lesssim 1$, play a much more important role. This is not the case here, since, as we shall see, at the transition line $y_{1} \approx \omega<1$.

Since our data are sufficiently close to the critical point, $p-p_{c}$ is small and thus we can take $u_{1} \sim\left(p-p_{c}\right)$. Moreover, also the product $\left(p-p_{c}\right) L^{y_{1}}$ is small, so that we can expand $f_{R}(x)$ in powers of $x$. Thus, we fit the numerical data to

$$
R=R^{*}+\sum_{n=1}^{n_{\max }} a_{n}\left(p-p_{c}\right)^{n} L^{n y_{1}}
$$

keeping $R^{*}$, the coefficients $\left\{a_{n}\right\}, p_{c}$, and $y_{1}$ as free parameters. Here we neglect scaling corrections. To monitor their role, we repeat the fits several times, each time only including data satisfying $L \geq L_{\min }$. For a given $L_{\min }, \chi^{2} / \mathrm{DOF}$ (DOF is the number of degrees of freedom of the fit) changes significantly as we increase $n_{\max }$ from 1 to 2 , and only marginally as we change this parameter from 2 to 3 . This indicates that the range of values of $p$ we are considering is too large to allow for a linear approximation of the scaling function $f_{R}(x)$. Instead, a quadratic approximation seems to be accurate enough. Thus, the results we present below correspond to $n_{\max }=2$.

In Table 1 we give the estimates of $R^{*}, p_{c}$, and $y_{1}$ from combined fits of $R_{\xi}, U_{4}$, and $U_{22}$. All quantities, except $y_{1}$, show a significant-much larger than the statistical errors-variation with $L_{\min }$. Moreover, the $\chi^{2}$ is very large. Clearly, scaling corrections are not negligible. In order to take them into account, we fit the $\mathrm{MC}$ data to

$$
R=R^{*}+\sum_{n=1}^{n_{\max }} a_{n}\left(p-p_{c}\right)^{n} L^{n y_{1}}+L^{-\omega} \sum_{k=0}^{k_{\max }} b_{k}\left(p-p_{c}\right)^{k} L^{k y_{1}},
$$

taking $\omega$ as a free parameter. Results for $k_{\max }=1$ and $n_{\max }=2$ are also reported in Table 1 . The $\chi^{2}$ is now significantly smaller and $\chi^{2} / \mathrm{DOF} \approx 1$, indicating that the fitting form (12)

Table 1 Estimates obtained from the analysis of the data at $\beta=2$. Above we report the results of the combined fits of $R_{\xi}, U_{4}$, and $U_{22}$ to (11) with $n_{\max }=2$. Below we report the results of the fits to (12) with $n_{\max }=2$ and $k_{\max }=1$

\begin{tabular}{rlllllll}
\hline$L_{\min }$ & $\chi^{2} / \mathrm{DOF}$ & $\omega$ & $R_{\xi}^{*}$ & $U_{4}^{*}$ & $U_{22}^{*}$ & $p_{c}$ & $y_{1}$ \\
\hline 8 & $9846 / 166$ & & $1.1865(3)$ & $1.09028(5)$ & $0.07287(4)$ & $0.892163(5)$ & $0.674(3)$ \\
12 & $3689 / 130$ & & $1.1996(4)$ & $1.08867(6)$ & $0.07293(5)$ & $0.892294(6)$ & $0.678(4)$ \\
16 & $1522 / 94$ & & $1.2068(4)$ & $1.08792(8)$ & $0.07327(7)$ & $0.892348(8)$ & $0.673(6)$ \\
24 & $441 / 61$ & & $1.2129(8)$ & $1.08734(12)$ & $0.07355(11)$ & $0.892389(11)$ & $0.676(8)$ \\
32 & $96 / 25$ & & $1.2172(16)$ & $1.08695(22)$ & $0.07350(20)$ & $0.892431(19)$ & $0.661(21)$ \\
& & & & & & & \\
8 & $212 / 159$ & $0.58(4)$ & $1.265(5)$ & $1.0810(6)$ & $0.0724(2)$ & $0.89265(2)$ & $0.677(17)$ \\
12 & $96 / 123$ & $0.64(8)$ & $1.249(6)$ & $1.0836(7)$ & $0.0741(3)$ & $0.89254(3)$ & $0.667(22)$ \\
16 & $77 / 87$ & $0.63(13)$ & $1.246(9)$ & $1.0842(9)$ & $0.0746(5)$ & $0.89251(3)$ & $0.660(30)$ \\
24 & $48 / 54$ & $0.50(43)$ & $1.254(38)$ & $1.0840(37)$ & $0.0752(25)$ & $0.89251(11)$ & $0.46(12)$ \\
\hline
\end{tabular}


describes the data at the level of their statistical accuracy. The results are stable and the estimates for $L_{\min } \geq 12$ are consistent within errors. These fits also provide an estimate of the correction-to-scaling exponent $\omega$. We find

$$
\omega=0.6(1)
$$

The estimates of $\omega$ and $y_{1}$ indicate that $\omega \approx y_{1}$, so that analytic and nonanalytic corrections behave analogously. Therefore, we should also consider the analytic corrections. For this purpose, we also performed fits to

$$
R=R^{*}+\sum_{n=1}^{n_{\max }} a_{n}\left[1+c\left(p-p_{c}\right)\right]^{n}\left(p-p_{c}\right)^{n} L^{n y_{1}},
$$

which corresponds to including the quadratic term in the expansion of the nonlinear scaling field $u_{1}$. The parameter $c$ is a new fitting parameter which is independent of the quantity one is analyzing. Fits to (14) are substantially equivalent to those to (11). For instance, the $\chi^{2}$ of the combined fit for $L_{\min }=8$ is 9846 , which is identical to that reported in Table 1 for the same value of $L_{\min }$. The coefficient $c$ is small and we estimate $|c| \lesssim 0.3$. Since our data satisfy $\left|p-p_{c}\right| \leq 0.0030$, the analytic term gives a tiny correction and does not influence the fit results.

Comparing the results of the different fits we arrive at the final estimates

$$
\begin{aligned}
y_{1} & =0.67(2), \quad v=1 / y_{1}=1.50(4), \\
p_{c} & =0.8925(1), \\
R_{\xi}^{*} & =1.25(3), \\
U_{4}^{*} & =1.084(3), \\
U_{22}^{*} & =0.074(1) .
\end{aligned}
$$

The central value corresponds to the result of the fit to (12) with $L_{\min }=12$; the errors are such to include the results of the fits to (11) and $L_{\min }=32$, and should take into account the systematic error due to further scaling corrections which have been neglected in our analyses.

We repeat the same type of analysis at $\beta=1.55$. We report in Table 2 the results of the fits to (11) and (12). In the latter case the data do not allow us to perform fits in which $\omega$ is a free parameter. Thus, we only report results of fits in which $\omega$ is fixed to $0.4,0.6$, and 0.8 , consistently with the estimate $\omega \approx 0.6$ presented above. Fits without scaling corrections are characterized by large values of $\chi^{2} / \mathrm{DOF}$ and by a systematic trend of the results. Fits with scaling corrections are significantly better. The estimates of $y_{1}$ and $U_{22}^{*}$ are in perfect agreement with those obtained at $\beta=2$. Those of $U_{4}^{*}$ and $R_{\xi}^{*}$ are substantially consistent: the difference between the estimates (17), (18) and the results of the fit with $\omega=0.6$-this is the fit which, in principle, should be more reliable - is of the order of two error bars and can thus be explained by the presence of residual scaling corrections which are not taken into account in our error estimate. Therefore, our analyses of the renormalized couplings are consistent with a critical transition line whose nature is $T$ independent: for $T<T^{*}$, the PF transition belongs to a unique universality class.

The estimate (15) is different from the Ising value $v=1$. Therefore, the PF fixed point associated with the transitions along the line $T<T^{*}$ is a new one, clearly distinct from 
Table 2 Estimates obtained from the analysis of the data at $\beta=1.55$. Above we report the results of the combined fits of $R_{\xi}, U_{4}$, and $U_{22}$ to (11) with $n_{\max }=2$. Below we report the results of fits to (12) with $n_{\max }=2, k_{\max }=1$, and $\omega$ fixed to $0.4,0.6,0.8$

\begin{tabular}{clllllll}
\hline$L_{\min }$ & $\omega$ & $\chi^{2} / \mathrm{DOF}$ & $R_{\xi}^{*}$ & $U_{4}^{*}$ & $U_{22}^{*}$ & $p_{c}$ & $y_{1}$ \\
\hline 8 & & $2099 / 88$ & $1.1362(8)$ & $1.0984(1)$ & $0.0756(1)$ & $0.89107(2)$ & $0.580(10)$ \\
12 & & $1045 / 73$ & $1.1457(8)$ & $1.0969(1)$ & $0.0753(1)$ & $0.89122(2)$ & $0.626(12)$ \\
16 & & $556 / 58$ & $1.1508(10)$ & $1.0962(2)$ & $0.0753(1)$ & $0.89128(2)$ & $0.636(13)$ \\
24 & & $236 / 43$ & $1.1543(15)$ & $1.0959(3)$ & $0.0755(2)$ & $0.89131(3)$ & $0.623(17)$ \\
& & & & & & & \\
8 & 0.4 & $77 / 82$ & $1.213(3)$ & $1.0878(5)$ & $0.0739(4)$ & $0.89160(3)$ & $0.663(52)$ \\
8 & 0.6 & $88 / 82$ & $1.190(2)$ & $1.0908(4)$ & $0.0744(3)$ & $0.89153(2)$ & $0.662(37)$ \\
8 & 0.8 & $111 / 82$ & $1.178(2)$ & $1.0904(3)$ & $0.0746(2)$ & $0.89148(2)$ & $0.659(29)$ \\
\hline
\end{tabular}

the Ising one, which controls the critical behavior for weak disorder. Analogously, our estimates of the critical value of the renormalized couplings differ from the Ising values [11, 59] $R_{\xi}^{*}=0.9050488292(4), U_{4}^{*}=1.167923(5), U_{22}^{*}=0$, and from those at the MNP (see Appendix C), which are $R_{\xi}^{*}=0.997(1), U_{4}^{*}=1.1264(4)$, and $U_{22}^{*}=0.0817(3)$.

Our analyses also give an estimate of $p_{c}$ for $\beta=1.55$ :

$$
p_{c}=0.8915(2) \text {. }
$$

Therefore, for both values of $\beta$ we find $p_{c}>p^{*}=0.89083(3)$. Thus, the PF transition line is reentrant, contradicting the conjecture of $[4,34,35,39]$.

\subsection{The Exponent $\eta$}

We determine the critical exponent $\eta$ from the critical behavior of the susceptibility $\chi$. As discussed in [58] in the context of the three-dimensional paramagnetic-glassy transition, close to the critical point the susceptibility $\chi$ behaves as

$$
\chi=\bar{u}_{h}^{2} L^{2-\eta} f_{\chi}\left[\left(p-p_{c}\right) L^{y_{1}}\right],
$$

where $\bar{u}_{h}$ is a function of $p$ related to the magnetic nonlinear scaling field. Note that we have approximated $u_{1}$ with $p-p_{c}$, because, as already discussed, the analytic dependence of the scaling field $u_{1}$ is negligible for our data.

Since we are very close to the critical point, we can expand all quantities in powers of $\left(p-p_{c}\right)$. For this reason we perform fits to

$$
\ln \chi=(2-\eta) \ln L+\sum_{n=0}^{n_{\max }} a_{n}\left(p-p_{c}\right)^{n} L^{n y_{1}}+\sum_{m=1}^{m_{\max }} b_{m}\left(p-p_{c}\right)^{m} .
$$

As before, we first analyze the data at $\beta=2$. To understand the role of the analytic corrections, we first perform fits of the data in which we fix $p_{c}=0.8925$ and $y_{1}=0.67$, which are the estimates obtained above. If we do not include the analytic correction (we set $b_{m}=0$ for any $m$ ) and we use $n_{\max }=2$, we obtain $\chi^{2} / \mathrm{DOF}=633 / 55,297 / 43$ from the analysis of the estimates of $\chi$ corresponding to lattices such that $L \geq L_{\min }=8,12$, respectively. If instead we include the analytic corrections taking $m_{\max }=1$, we obtain $\chi^{2} / \mathrm{DOF}=42 / 54,30 / 42$. 
Table 3 Estimates of $\eta$ from fits to (22) with $n_{\max }=2$ and $m_{\max }=1$. We fix $y_{1}=0.67(2)$ in both fits. The reported error takes into account the error bar on $y_{1}$ and on $p_{c}$ (for the fit in which this quantity is fixed). Analyses of the data at $\beta=2$

\begin{tabular}{rllllll}
\hline$L_{\min }$ & \multicolumn{2}{l}{$p_{c}=0.8925(1)$} & & \multicolumn{2}{l}{$p_{c}$ free parameter } \\
\cline { 2 - 3 } & $\chi^{2} / \mathrm{DOF}$ & $\eta$ & & $\chi^{2} / \mathrm{DOF}$ & $\eta$ & $p_{c}$ \\
\hline 8 & $42 / 54$ & $0.1235(13)$ & $41 / 53$ & $0.1236(4)$ & $0.89249(2)$ \\
12 & $30 / 42$ & $0.1235(15)$ & $28 / 41$ & $0.1241(5)$ & $0.89245(4)$ \\
16 & $21 / 30$ & $0.1234(16)$ & $20 / 29$ & $0.1243(8)$ & $0.89244(5)$ \\
24 & $15 / 19$ & $0.1233(20)$ & $14 / 18$ & $0.1254(20)$ & $0.89238(11)$ \\
32 & $8 / 7$ & $0.1232(23)$ & $7 / 6$ & $0.1278(40)$ & $0.89227(20)$
\end{tabular}

The improvement is clearly significant, indicating that the analytic corrections cannot be neglected.

In Table 3 we report the results of the fits corresponding to $n_{\max }=2$ and $m_{\max }=1$. In all cases we fix $y_{1}$ to $0.67(2)$, as indicated by (15). This is not crucial, since the estimates of the exponent $\eta$ are quite insensitive to this parameter. The results show instead a significant dependence on $p_{c}$ and thus, we present fits in which $p_{c}$ is fixed to the value (16) and fits in which $p_{c}$ is a free parameter. The estimates of the two fits are substantially consistent and show a tiny dependence on $L_{\min }$. Also the estimates of $p_{c}$ are consistent with the value (16).

We also considered nonanalytic scaling corrections, performing a fit of the form

$$
\ln \chi=(2-\eta) \ln L+\sum_{n=0}^{n_{\max }} a_{n}\left(p-p_{c}\right)^{n} L^{n y_{1}}+\sum_{m=1}^{m_{\max }} b_{m}\left(p-p_{c}\right)^{m}+c L^{-\omega} .
$$

We fix $p_{c}=0.8925, y_{1}=0.67, \omega=0.6, n_{\max }=2, m_{\max }=1$, and obtain $\eta=0.1234(13)$, $c=0.000$ (3) for $L_{\min }=8$ : there is no evidence of nonanalytic scaling corrections.

To avoid the use of $p_{c}$, note that (9) can be inverted to give $u_{1} L^{y_{1}} \approx\left(p-p_{c}\right) L^{y_{1}}$ as a function of $R$. Thus, (21) can also be rewritten as

$$
\chi=\bar{u}_{h}^{2} L^{2-\eta} g_{\chi}(R)\left[1+O\left(L^{-\omega}\right)\right],
$$

where $R$ is a renormalized coupling. A polynomial approximation for $\bar{u}_{h}(p)$ and $g_{\chi}(R)$ gives the fitting form

$$
\ln \chi=(2-\eta) \ln L+\sum_{n=0}^{n_{\max }} a_{n} R^{n}+\sum_{m=1}^{m_{\max }} b_{m} p^{m}
$$

Fits to this form have a quite large $\chi^{2}$, which is not unexpected since we already found that the renormalized couplings show significant scaling corrections. Moreover, the results depend significantly on the minimum lattice size $L_{\min }$ of the data included in the fit. Scaling corrections must therefore be included. We thus consider

$$
\ln \chi=(2-\eta) \ln L+\sum_{n=0}^{n_{\max }} a_{n} R^{n}+\sum_{m=1}^{m_{\max }} b_{m} p^{m}+L^{-\omega} \sum_{k=0}^{k_{\max }} c_{k} R^{k} .
$$

The results of these fits are reported in Table 4. The $\chi^{2}$ is good; moreover, the results do not depend on which quantity is used in the fit, are stable with $L_{\min }$, and are consistent with those reported in Table 3.

Analogous analyses can be performed at $\beta=1.55$. Also in this case the analytic corrections cannot be neglected and thus we only consider fits with $m_{\max }=1$. The results of the 
Table 4 Estimates of $\eta$ from fits to (26) with $n_{\max }=2, m_{\max }=1$, $k_{\max }=0, \omega$ free parameter. On the left we use $R=U_{4}$, on the right we use $R=R_{\xi}$. Analyses of the data at $\beta=2$

\begin{tabular}{rlllll}
\hline$L_{\min }$ & $U_{4}$ & & & $R \xi$ \\
\cline { 2 - 3 } \cline { 6 - 6 } & $\chi^{2} / \mathrm{DOF}$ & $\eta$ & & $\chi^{2} / \mathrm{DOF}$ & $\eta$ \\
\hline 8 & $72 / 52$ & $0.1247(5)$ & $47 / 52$ & $0.1245(14)$ \\
12 & $57 / 40$ & $0.1250(8)$ & $40 / 40$ & $0.1253(22)$ \\
16 & $54 / 28$ & $0.1255(9)$ & $36 / 28$ & $0.1255(48)$ \\
\hline
\end{tabular}

\begin{tabular}{rllllll}
\hline$L_{\min }$ & \multicolumn{2}{l}{$p_{c}=0.8915(2)$} & & \multicolumn{2}{l}{$p_{c}$ free parameter } \\
\cline { 2 - 3 } \cline { 6 - 7 } & $\chi^{2} / \mathrm{DOF}$ & $\eta$ & & $\chi^{2} / \mathrm{DOF}$ & $\eta$ & $p_{c}$ \\
\hline 8 & $25 / 28$ & $0.1336(26)$ & $23 / 27$ & $0.1342(7)$ & $0.89145(3)$ \\
12 & $23 / 23$ & $0.1335(30)$ & $22 / 22$ & $0.1341(9)$ & $0.89145(4)$ \\
16 & $21 / 18$ & $0.1335(33)$ & $20 / 17$ & $0.1341(12)$ & $0.89146(6)$ \\
24 & $16 / 13$ & $0.1330(38)$ & $16 / 12$ & $0.1315(23)$ & $0.89158(12)$ \\
32 & $10 / 8$ & $0.1330(42)$ & $10 / 7$ & $0.1361(46)$ & $0.89133(21)$ \\
\hline
\end{tabular}

Table 5 Estimates of $\eta$ from fits to (22) with $n_{\max }=2$ and $m_{\max }=1$. We fix $y_{1}=0.67(2)$ in both fits. The reported error takes into account the error bar on $y_{1}$ and on $p_{c}$ (for the fit in which this quantity is fixed). Analyses of the data at $\beta=1.55$

fits to (22) are reported in Table 5. The dependence of the results on $L_{\min }$ is tiny. Moreover, the estimates of $p_{c}$ obtained in the analyses in which this quantity is a free parameter are perfectly consistent with the estimate (20). Similar, though less stable, results are obtained by fitting the data to (26). We fix $\omega=0.6(1)$ as in the case of the analyses of the renormalized couplings. For $L_{\min }=8$ we obtain $\eta=0.1304(5)$ and $\eta=0.1340(5)$ by using $U_{4}$ and $R_{\xi}$, respectively; for $L_{\min }=12$ we obtain instead $\eta=0.1317(8)$ and $\eta=0.1329(8)$.

Collecting all results, from the analyses of the data at $\beta=2$ we would estimate $\eta=$ 0.125 (3). The analyses at $\beta=1.55$ give a slightly different value, $\eta=0.132(4)$. The difference is tiny-less than two combined error bars-but indicates that there are corrections which are not fully taken into account by our analyses. As final estimate we report the average of the two results,

$$
\eta=0.128(8) \text {. }
$$

The error we quote is quite conservative and essentially includes the estimates of all fits for both values of $\beta$.

\subsection{The Critical Exponent $\beta$ and a Check of Hyperscaling}

The critical exponent $\beta$ (not to confused with the inverse temperature) can be determined from the critical behavior of the magnetization. $\mathrm{RG}$ predicts

$$
m=\bar{u}_{h} L^{-\beta / v} f_{m}\left[\left(p-p_{c}\right) L^{y_{1}}\right]
$$

where $\bar{u}_{h}$ is the same function which appears in (21) and $f_{m}(x)$ is a universal function. Expanding this scaling relation around the critical point we obtain the fitting form

$$
\ln m=-\frac{\beta}{v} \ln L+\sum_{n=0}^{n_{\max }} a_{n}\left(p-p_{c}\right)^{n} L^{n y_{1}}+\sum_{m=1}^{m_{\max }} b_{m}\left(p-p_{c}\right)^{m} .
$$

As before we fix $n_{\max }=2, m_{\max }=1$, and use the best available estimates of $p_{c}$. For $\beta=2$ a fit of the data satisfying $L \geq L_{\min }=24$ gives $\beta / v=0.0613(11)$; for $L_{\min }=32$ we obtain 
Fig. 3 The specific heat at $\beta=2$ and $p=0.8925 \approx p_{c}$ versus $L^{-2 / 3}$. The dashed line is obtained by fitting the MC data to $a+b L^{-2 / 3}$

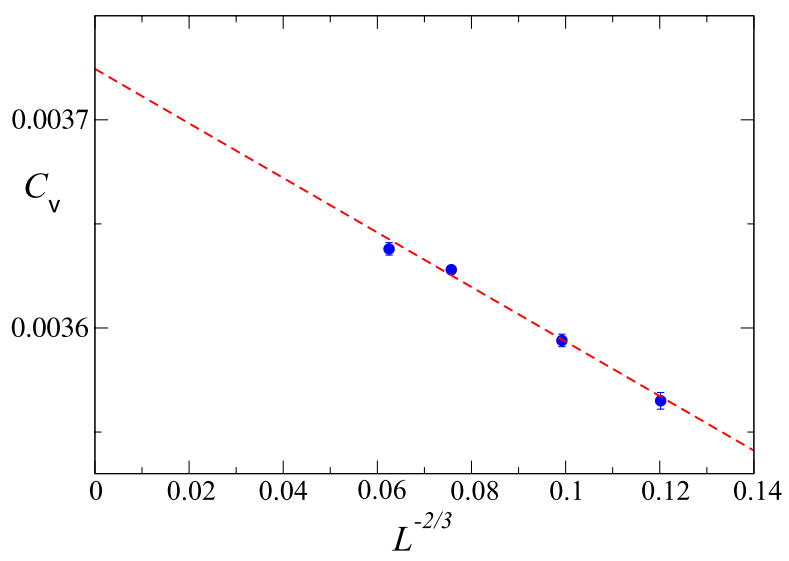

instead $\beta / v=0.0614(12)$. For $\beta=1.55$ and $L_{\min }=32$ we obtain $\beta / v=0.0661(22)$. As in the case of $\eta$, we observe a tiny difference between the estimates obtained at the two values of the temperature. It probably indicates the presence of additional scaling corrections which are not taken into account by our scaling Ansatz. A conservative estimate of the critical exponent which is consistent with all results is

$$
\frac{\beta}{v}=0.063(3) \quad \beta=0.095(5) .
$$

We can now check hyperscaling. If it holds, we should have $2 \beta / v-\eta+2=d=2$. We find

$$
\frac{2 \beta}{v}-\eta+2=2.00(1)
$$

Hyperscaling is verified quite precisely.

Finally, we consider the specific heat. At $p=p_{c}$ we expect

$$
C_{v}=a+b L^{\alpha / v},
$$

where $a$ is due to the analytic contribution to the free energy. If hyperscaling holds, we should have $\alpha=2-2 v$, so that

$$
\frac{\alpha}{v}=\frac{2}{v}-2=2 y_{1}-2=-0.66(4) .
$$

A precise determination of $\alpha / v$ from the data is quite difficult, because $\alpha / v<0$-the singular part decreases as $L \rightarrow \infty$. Thus, we have only checked that our data are consistent with hyperscaling. In Fig. 3 we show the specific heat for $\beta=2$ and $L \geq 24$ versus $L^{-2 / 3}$. The results are consistent, supporting hyperscaling.

\subsection{The Derivative $d p_{c} / d \beta$}

As a final test of our results we consider the derivative with respect to $\beta$ of a renormalized coupling $R$. In the FSS limit $R$ behaves as

$$
R=f_{R}\left(u_{1} L^{y_{1}}\right)+u_{\omega} L^{-\omega} f_{R, \omega}\left(u_{1} L^{y_{1}}\right)+\cdots
$$


where the scaling fields $u_{1}$ and $u_{\omega}$ are functions of the system parameters, hence of $\beta$ and $p$. Moreover, $u_{1}$ vanishes on the critical line. From (34) we obtain

$$
\frac{\partial R}{\partial \beta}=\frac{\partial u_{1}}{\partial \beta} L^{y_{1}}\left[f_{R}^{\prime}\left(u_{1} L^{y_{1}}\right)+u_{\omega} L^{-\omega} f_{R, \omega}^{\prime}\left(u_{1} L^{y_{1}}\right)\right]+\frac{\partial u_{\omega}}{\partial \beta} L^{-\omega} f_{R, \omega}\left(u_{1} L^{y_{1}}\right)+\cdots .
$$

If the critical value $p_{c}$ is $\beta$ independent, $\partial u_{1} / \partial \beta$ vanishes on the critical line, so that $\partial R / \partial \beta$ behaves as $L^{-\omega}$ for $L \rightarrow \infty$, i.e. the derivative vanishes in the critical large- $L$ limit. This is not surprising, since for $p=p_{c}=p^{*}$ and any $\beta$ we would have $R=R^{*}+O\left(L^{-\omega}\right)$, with $R^{*}$ independent of $\beta$. On the other hand, if the transition is reentrant, $\partial R / \partial \beta$ diverges as $L^{y_{1}}$.

We have checked the validity of (35) by using the data at $\beta=2$. The fits of the renormalized couplings $R$ give us estimates of the expansion of $R$ around $p_{c}$. In particular, fits to (12) give us estimates of the coefficients $a_{n}$. We have thus fitted $\partial R / \partial \beta$ to the following expression:

$$
\frac{\partial R}{\partial \beta}=k_{0} L^{y_{1}}\left[a_{1}+2 a_{2}\left(p-p_{c}\right) L^{y_{1}}\right]+k_{1} L^{y_{1}-\omega} .
$$

We take $y_{1}=0.67(2), p_{c}=0.8925(1), \omega=0.6(1)$, and $a_{1}$ and $a_{2}$ from the fits of $R$ to (12); $k_{0}$ and $k_{1}$ are free parameters. The estimates of $k_{0}$ do not vary significantly with $L_{\min }$. Moreover, results obtained by using $\partial R_{\xi} / \partial \beta$ and $\partial U_{4} / \partial \beta$ are fully consistent. Comparing all results we obtain the estimate

$$
k_{0}=-0.0020(3)
$$

To interpret this result, note that (9), (10), and (12) allow us to identify

$$
a_{1}=A_{0}(\beta) f_{R}^{\prime}(0)
$$

Instead, comparing (36) with (35) we obtain

$$
k_{0} a_{1}=\left.\frac{\partial u_{1}}{\partial \beta}\right|_{p_{c}} f_{R}^{\prime}(0) .
$$

Now, (10) gives

$$
\left.\frac{\partial u_{1}}{\partial \beta}\right|_{p_{c}}=-A_{0}(\beta) \frac{d p_{c}}{d \beta} .
$$

It follows

$$
\frac{d p_{c}}{d \beta}=-k_{0}=0.0020(3) .
$$

Again, this result shows that the transition is reentrant. It is also consistent with the crude estimate

$$
\frac{d p_{c}}{d \beta} \approx \frac{p_{c}(2)-p_{c}(1.55)}{2-1.55} \approx 0.0022
$$

\section{Conclusions}

In this paper we have studied the nature of the transition line which starts from the MNP and ends at $T=0$ and which separates the paramagnetic phase from the ferromagnetic phase. 
For this purpose, we have presented FSS analyses of MC data on lattices of linear size $L$ up to $L=64$ for $\beta \equiv 1 / T=2$ and $\beta=1.55$.

Our main results are the following.

(i) The PF transition line below the MNP is reentrant. Indeed, we find $p_{c}=0.8915(2)$ at $T=1 / 1.55 \approx 0.645$ and $p_{c}=0.8925(1)$ at $T=0.5$. Therefore, $p_{c}>p^{*}=0.89083(3)$ for any $T<T^{*}=0.9527(1)$, where $X^{*}=\left(T^{*}, p^{*}\right)$ is the location of the MNP.

(ii) The PF transitions are of second order with a standard power-law behavior.

(iii) The estimated values of the critical exponents and of the large- $L$ limit of the RG invariant quantities $U_{4}, R_{\xi}$, and $U_{22}$ at two different points of the line $(\beta=1.55$ and $\beta=2)$ suggest that the PF transitions for $0<T<T^{*}$ belong to a unique universality class. In particular, the corresponding critical exponents are

$$
v=1.50(4), \quad \eta=0.128(8), \quad \beta=0.095(5) .
$$

They satisfy the hyperscaling relation $2 \beta / v-\eta=d-2=0$. Our MC data are also consistent with the hyperscaling relation $\alpha=2-d v=2-2 v$, which gives $\alpha=-1.00(8)$. Using the scaling relation $\gamma=(2-\eta) \nu$, we derive $\gamma=2.81(8)$. The estimates (43) are definitely different from the Ising values $v=1, \eta=1 / 4, \beta=1 / 8$. We note that they are consistent with the simple rational expressions $v=3 / 2, \eta=1 / 8$.

(iv) The above results show that in two dimensions there are two fixed points which control the PF transitions in disordered random-bond Ising systems: besides the standard Ising fixed point, which is relevant for small disorder and controls the critical behavior along the PF transition line for $T^{*}<T \leq T_{\text {Is }}$, there is also a strong-disorder fixed point which controls the critical behavior along the PF transition line for $0<T<T^{*}$. The resulting phase diagram is consistent with the results of [30,43]. Note that frustration and not simply disorder is the relevant property, which gives rise to the new fixed point. Indeed, in randomly-dilute Ising systems, in which there is dilution but not frustration, there is no evidence of a new strong-disorder fixed point [11].

It is interesting to compare our results with those obtained at $T=0$. McMillan [43] extrapolated the RG results to $T=0$ (this is correct under the assumption that the limit $T \rightarrow 0$ is regular) and obtained $v=1.42(8)$. Wang et al. [45] obtained $v=1.46(1)$ from the scaling of the failure probability. Amoruso and Hartmann [44] found $v=1.55(1)$ from the analysis of the Binder cumulant and the magnetization exponent $\beta=0.09(1){ }^{2}$ They also analyzed the domain-wall energy, obtaining $\Delta E=L^{\rho} f\left(\left(p-p_{0}\right) L^{y_{1}}\right)$ with $\rho=0.12(5)$ and $y_{1}=0.75(5)$. This gives $v=1 / y_{1}=1.33(9)$. The exponent $\eta$ associated with the spinspin correlation has been estimated in [18], obtaining $\eta \approx 0.13$. Note that these estimates are consistent with the hyperscaling relation $2 \beta / v-\eta=d-2=0$. Therefore, even if these results refer to a $T=0$ transition, the magnetic exponents are consistent with hyperscaling.

The $T=0$ results are very close to ours. Note also that, at $T=0$, the relation $U_{4}^{*}=$ $U_{22}^{*}+1$ holds. $^{3}$ This relation is approximately satisfied by our finite- $T$ data, see (18) and (19); the slight discrepancy might be due to the presence of neglected additional scaling corrections. All results are therefore consistent with a single magnetic fixed point that controls

\footnotetext{
${ }^{2}$ Amoruso and Hartmann [44] report $\beta=0.9(1)$. Alexander Hartmann communicated to us that the correct result is $\beta=0.09$ (1).

${ }^{3}$ Indeed, assuming a nondegenerate ground state (this should be the case in two dimensions), we have $\mu_{4}=$ $\mu_{2}^{2}$ (see Appendix B for the definitions), from which the relation follows.
} 
Fig. 4 The phase diagram of the square-lattice $\pm J$ Ising model. The estimates of the critical points for $T>T^{*}$ are taken from $[11,22]$. The estimate of the $T=0$ transition point is taken from [44, 45]. The dashed lines are interpolations discussed in Appendix C, while the dotted line starting at the Ising point corresponds to the approximation (3)

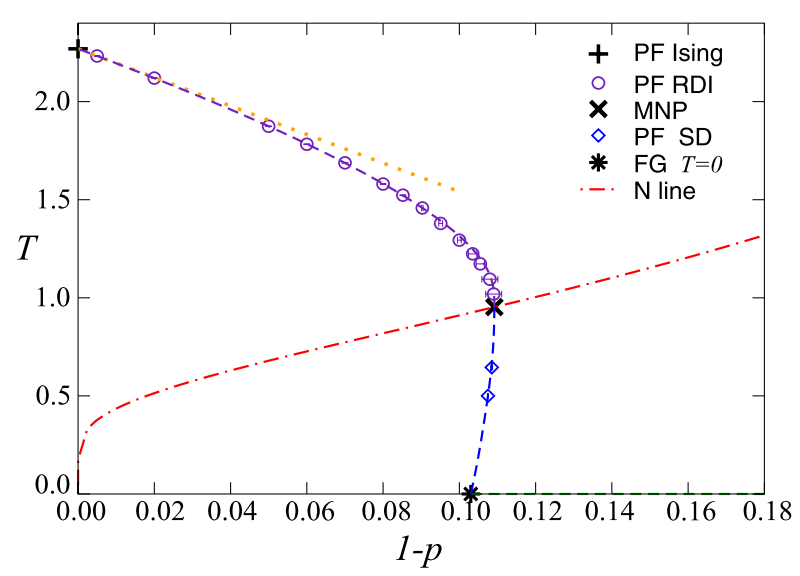

the magnetic critical behavior both at $T>0$ and at $T=0$. At the multicritical $T=0$ point, glassy and magnetic modes are apparently effectively decoupled.

Finally, we have improved the estimates of the critical parameters at the MNP by a new FSS analysis of MC simulations up to $L=64$ along the $\mathrm{N}$ line. We obtain $p^{*}=0.89083$ (3) and $T^{*}=0.9527(1), y_{1}=0.66(1)$ and $y_{2}=0.250(2)$ for the RG dimensions of the two relevant operators in the absence of external field, and $\eta=0.177(2)$ for the magnetic critical exponent associated with the spin-spin correlation function.

In Fig. 4 we report the available estimates of the critical points and report simple interpolations, discussed in Appendix $\mathrm{C}$, which take into account all theoretical predictions and numerical results.

Acknowledgements Discussions with Marco Picco and correspondence with Nihat Berker and Alexander Hartmann are gratefully acknowledged.

\section{Appendix A: Some Details on the Monte Carlo Simulations}

In our parallel-tempering simulations we consider $N_{T}$ systems at the same value of $p$ and at $N_{T}$ different inverse temperatures $\beta_{\min } \equiv \beta_{1}, \ldots, \beta_{N_{T}} \equiv \beta_{\max }$, where $\beta_{\max }$ is chosen to be either 2 or 1.55 . To avoid repeating the runs twice, for $L=48$ and $64, \beta_{\max }$ is always chosen to be 2 , while one of the $\beta_{i}$ corresponds to 1.55 . Moreover, for all values of $L$, we choose $\beta_{i}=\beta_{N}(p)$ for some $i$, where $\beta_{N}(p)$ is given in (4), so that the corresponding point lies on the $\mathrm{N}$ line. This choice gives us estimates along the $\mathrm{N}$ line, which can be compared with exact and previous numerical results. They provide a check of the numerical simulations and allow us to improve the estimates of the critical parameters of [15], see Appendix C.

The elementary unit of the algorithm consists in $N_{\mathrm{ex}}=20$ Metropolis sweeps for each configuration followed by an exchange move. We consider all pairs of configurations corresponding to nearby temperatures and propose a temperature exchange with acceptance probability

$$
\mathcal{P}=\exp \left\{\left(\beta_{i}-\beta_{i+i}\right)\left(E_{i}-E_{i+1}\right)\right\},
$$

where $E_{i}$ is the energy of the system at inverse temperature $\beta_{i}$. We generate $N_{s}$ disorder samples, and for every sample we perform a MC run of $N_{\text {run }}$ Metropolis sweeps for each $\beta_{i}$ value. The first $N_{\text {therm }}$ iterations are discarded for thermalization (see [15] for a discussion of 
Table 6 Parameters of the random-exchange $\mathrm{MC}$ runs for $L \geq 32$

\begin{tabular}{llllrlll}
\hline$L$ & $p$ & $\beta_{\min }$ & $\beta_{\max }$ & $N_{T}$ & $N_{\mathrm{s}} / 64$ & $N_{\text {run }} / 10^{3}$ & $N_{\text {therm }} / 10^{3}$ \\
\hline 32 & 0.8910 & 0.800 & 1.55 & 5 & 15625 & 800 & 240 \\
32 & 0.8915 & 0.800 & 1.55 & 5 & 15625 & 800 & 240 \\
32 & 0.8920 & 0.800 & 1.55 & 5 & 15625 & 800 & 240 \\
32 & 0.8925 & 0.800 & 1.55 & 5 & 15625 & 800 & 240 \\
32 & 0.8930 & 0.800 & 1.55 & 5 & 15625 & 800 & 400 \\
32 & 0.8915 & 0.800 & 2 & 6 & 15625 & 800 & 240 \\
32 & 0.8920 & 0.800 & 2 & 6 & 15625 & 800 & 240 \\
32 & 0.8925 & 0.800 & 2 & 6 & 15625 & 800 & 240 \\
32 & 0.8930 & 0.800 & 2 & 6 & 15625 & 800 & 240 \\
48 & 0.8915 & 0.740 & 2 & 9 & 31250 & 2000 & 400 \\
48 & 0.8920 & 0.740 & 2 & 9 & 31250 & 2000 & 400 \\
48 & 0.8925 & 0.740 & 2 & 9 & 31250 & 2000 & 400 \\
48 & 0.8930 & 0.740 & 2 & 9 & 31250 & 2000 & 600 \\
64 & 0.8915 & 0.710 & 2 & 13 & 7813 & 3000 & 900 \\
64 & 0.8920 & 0.710 & 2 & 13 & 7813 & 3000 & 900 \\
64 & 0.8925 & 0.710 & 2 & 13 & 7813 & 3000 & 900 \\
64 & 0.8930 & 0.710 & 2 & 13 & 7813 & 3000 & 900 \\
\hline
\end{tabular}

the thermalization issues). The parameters of the runs with $L \geq 32$ are reported in Table 6 . Finally, note that the determination of $U_{22}$ requires the computation of a disorder average of the square of a thermal average. We use an essentially bias-free estimator discussed in [60].

\section{Appendix B: Definitions}

The two-point correlation function is defined as

$$
G(x) \equiv\left[\left\langle\sigma_{0} \sigma_{x}\right\rangle\right]
$$

where the angular and the square brackets indicate the thermal average and the quenched average over disorder, respectively. We define the magnetic susceptibility $\chi \equiv \sum_{x} G(x)$ and the correlation length $\xi$,

$$
\xi^{2} \equiv \frac{\widetilde{G}(0)-\widetilde{G}\left(q_{\min }\right)}{\hat{q}_{\min }^{2} \widetilde{G}\left(q_{\min }\right)},
$$

where $q_{\min } \equiv(2 \pi / L, 0), \hat{q} \equiv 2 \sin q / 2$, and $\widetilde{G}(q)$ is the Fourier transform of $G(x)$. We also consider the magnetization $m$ defined as

$$
m=\frac{1}{V}\left[\left\langle\left|\sum_{x} \sigma_{x}\right|\right\rangle\right],
$$

where $V$ is the volume, and the specific heat $C_{v}$

$$
C_{v}=\frac{1}{V}\left[\left\langle\mathcal{H}^{2}\right\rangle-\langle\mathcal{H}\rangle^{2}\right],
$$


where $\mathcal{H}$ is the Hamiltonian.

We also consider quantities (we call them renormalized couplings) that are invariant under RG transformations in the critical limit. Beside the ratio

$$
R_{\xi} \equiv \xi / L
$$

we consider the RG invariant quantities

$$
U_{4} \equiv \frac{\left[\mu_{4}\right]}{\left[\mu_{2}\right]^{2}}, \quad U_{22} \equiv \frac{\left[\mu_{2}^{2}\right]-\left[\mu_{2}\right]^{2}}{\left[\mu_{2}\right]^{2}}, \quad U_{d} \equiv U_{4}-U_{22},
$$

where

$$
\mu_{k} \equiv\left\langle\left(\sum_{x} \sigma_{x}\right)^{k}\right\rangle
$$

\section{Appendix C: Critical Exponents at the Multicritical Point}

In each parallel-tempering simulation we fixed $p$ and considered several values of $\beta$ from $\beta_{\min }<\beta^{*}$ up to $\beta_{\max }$ which was either 2 or 1.55 , hence larger than the multicritical value $\beta^{*}$. In all runs we were careful to include a point on the $\mathrm{N}$ line. Since the energy is known exactly on this line, this choice allowed us to test the correctness of the simulation code. Moreover, we were able to collect a significant amount of new data, which can be combined with the old ones presented in [15]. As we shall see, the FSS analyses of this new set of data allow us to improve the estimates of the critical parameters.

As in [15] we perform combined fits of the renormalized couplings to (11) and (12). The new results are reported in Tables 7 and 8 . The estimates of $y_{1}$ are quite stable and essentially independent of $L_{\min }$, of the observable, and of the scaling corrections. We thus quote

$$
y_{1}=0.66(1),
$$

where the error is chosen quite conservatively, and is such to include all results. This result is fully consistent with the estimate $y_{1}=0.655(15)$ of [15]. The estimates of $p^{*}$ vary between 0.89081 and 0.89086 , so that we quote

$$
p^{*}=0.89083(3)
$$

Table 7 Estimates of $p^{*}$ and $y_{1}$ at the MNP. Results from combined fits of three different renormalized couplings to (11) with $n_{\max }=2$. Here $U_{d} \equiv U_{4}-U_{22}$

\begin{tabular}{lllll}
\hline & $L_{\min }$ & $\chi^{2} / \mathrm{DOF}$ & $p^{*}$ & $y_{1}$ \\
\hline$R_{\xi}, U_{4}, U_{22}$ & 12 & $383 / 289$ & $0.890864(4)$ & $0.659(2)$ \\
& 16 & $207 / 220$ & $0.890844(4)$ & $0.658(2)$ \\
& 24 & $120 / 151$ & $0.890828(6)$ & $0.658(3)$ \\
& 32 & $58 / 82$ & $0.890822(8)$ & $0.651(5)$ \\
$R_{\xi}, U_{4}, U_{d}$ & 12 & $424 / 289$ & $0.890853(3)$ & $0.660(1)$ \\
& 16 & $248 / 220$ & $0.890856(4)$ & $0.660(2)$ \\
& 24 & $194 / 151$ & $0.890850(5)$ & $0.659(5)$ \\
& 32 & $100 / 82$ & $0.890848(7)$ & $0.653(5)$ \\
\hline
\end{tabular}


Table 8 Estimates of $p^{*}, y_{1}$, and $\omega$ at the MNP. Results from combined fits of three different renormalized couplings to (12) with $n_{\max }=2$ and $k_{\max }=1$

\begin{tabular}{llllll}
\hline & $L_{\min }$ & $\chi^{2} / \mathrm{DOF}$ & $p^{*}$ & $y_{1}$ & $\omega$ \\
\hline$R_{\xi}, U_{4}, U_{22}$ & 6 & $283 / 318$ & $0.890822(7)$ & $0.665(3)$ & $1.79(13)$ \\
& 8 & $232 / 300$ & $0.890814(9)$ & $0.660(10)$ & $1.98(25)$ \\
$R_{\xi}, U_{4}, U_{d}$ & 6 & $396 / 318$ & $0.890864(3)$ & $0.662(2)$ & $3.18(10)$ \\
& 8 & $332 / 300$ & $0.890857(4)$ & $0.660(2)$ & $4.41(27)$ \\
\hline
\end{tabular}

This estimate agrees with that we obtained in [15], i.e. $p^{*}=0.89081(7)$. Moreover, it is in full agreement with the recent calculations of [14]: Two different approximations gave $p^{*} \approx 0.890822$ and $p^{*} \approx 0.890813$.

Our analyses also provide estimates of the critical-point value of the renormalized couplings:

$$
\begin{aligned}
R_{\xi}^{*} & =0.997(1), \\
U_{4}^{*} & =1.1264(4), \\
U_{22}^{*} & =0.0817(3) .
\end{aligned}
$$

Scaling corrections are particularly weak and apparently decay as $L^{-2}$ or faster. Note that this does not necessarily imply the presence of nonanalytic corrections associated with RG irrelevant operators with $\omega \approx 2$. Indeed, in all cases we expect contributions due to the regular part of the free energy, which decay as $L^{\eta-2} \approx L^{-1.8}$.

The critical exponent $y_{2}$ is derived from the critical behavior of $R^{\prime} \equiv \partial R / \partial \beta$, where $R$ is a renormalized coupling [15]. Neglecting scaling correction, its FSS behavior is given by

$$
R^{\prime}=\frac{\partial u_{1}}{\partial \beta} L^{y_{1}} f_{1}\left(u_{1} L^{y_{1}}, u_{2} L^{y_{2}}\right)+\frac{\partial u_{2}}{\partial \beta} L^{y_{2}} f_{2}\left(u_{1} L^{y_{1}}, u_{2} L^{y_{2}}\right)
$$

where $u_{1}$ and $u_{2}$ are the nonlinear scaling fields associated with the two leading relevant operators. In general, we expect $[36,37] u_{2}$ to vanish on the $\mathrm{N}$ line, so that

$$
u_{2}(\beta, p)=S\left(\beta-\beta_{N}(p), p-p^{*}\right),
$$

where $\beta_{N}(p)=1 / T_{N}(p), T_{N}(p)$ is defined in (4), and the function $S(x, y)$ is such that $S(0, y)=0$ and $\partial S(0,0) / \partial x \neq 0$. Since the transition lines must be tangent to the line $p=$ $p^{*}$ as a consequence of a general rigorous inequality [13], we also have

$$
u_{1}(\beta, p)=p-p^{*}+\text { quadratic terms. }
$$

The independence of $u_{1}$ on $\beta$ at leading order, implies that the first term in (C.6) vanishes at the MNP, so that $R^{\prime} \sim L^{y_{2}}$ for $L \rightarrow \infty$ at $p=p^{*}$.

In order to compute $y_{2}$, we perform three different fits of our data on the $\mathrm{N}$ line. In the first one, we neglect the $p$ dependence of $\partial u_{2} / \partial \beta$ and set $\partial u_{1} / \partial \beta=0$. Then, setting $u_{2}=0$ and expanding in powers of $u_{1} L^{y_{1}} \sim\left(p-p^{*}\right) L^{y_{1}}$, we obtain

$$
\ln R^{\prime}=y_{2} \ln L+\sum_{n=0}^{n_{\max }} a_{n}\left(p-p^{*}\right)^{n} L^{n y_{1}} .
$$


Table 9 Estimates of $y_{2}$ at the MNP. We fix $y_{1}=0.66(1)$ and $p_{c}=0.89083(3)$. Results from fits of the derivative of the renormalized couplings $R_{\xi}$ and $U_{4}$. On the left the results refer to the fit to (C.9) with $n_{\max }=2$, on the right to the fit to (C.10) with $n_{\max }=2$ and $m_{\max }=1$. The error in parentheses is the sum of the statistical error and of the error due to uncertainty of $y_{1}$; the error in brackets gives the variation of the estimate as $p_{c}$ varies by one error bar

\begin{tabular}{rrllll}
\hline & $L_{\min }$ & $\chi^{2} / \mathrm{DOF}$ & $y_{2}$ & $\chi^{2} / \mathrm{DOF}$ & $y_{2}$ \\
\hline$R_{\xi}^{\prime}$ & 8 & $90 / 102$ & $0.2533(6)[5]$ & $57 / 101$ & $0.2521(5)[5]$ \\
& 12 & $88 / 96$ & $0.2535(7)[5]$ & $56 / 95$ & $0.2519(6)[6]$ \\
& 16 & $60 / 73$ & $0.2530(9)[6]$ & $45 / 72$ & $0.2514(8)[7]$ \\
& 24 & $37 / 50$ & $0.2531(13)[7]$ & $31 / 49$ & $0.2515(13)[8]$ \\
& 32 & $16 / 27$ & $0.2528(21)[8]$ & $15 / 26$ & $0.2523(21)[8]$ \\
$U_{4}^{\prime}$ & 8 & $158 / 102$ & $0.2492(9)[18]$ & $90 / 101$ & $0.2480(4)[17]$ \\
& 12 & $148 / 96$ & $0.2496(11)[19]$ & $89 / 95$ & $0.2478(5)[18]$ \\
& 16 & $95 / 73$ & $0.2450(14)[21]$ & $65 / 72$ & $0.2480(7)[21]$ \\
& 24 & $53 / 50$ & $0.2509(18)[25]$ & $42 / 49$ & $0.2490(11)[24]$ \\
& 32 & $32 / 27$ & $0.2514(23)[28]$ & $29 / 26$ & $0.2500(19)[27]$ \\
\hline
\end{tabular}

In the second fit we include the nontrivial dependence of $u_{2}$ on $\beta$ and $p$. We fit the results to

$$
\ln R^{\prime}=y_{2} \ln L+\sum_{n=0}^{n_{\max }} a_{n}\left(p-p^{*}\right)^{n} L^{n y_{1}}+\sum_{m=1}^{m_{\max }} b_{m}\left(p-p^{*}\right)^{m} .
$$

Finally, note that $u_{1}$ may depend on $\beta$ at quadratic and higher orders, so that on the $\mathrm{N}$ line one may have

$$
\frac{\partial u_{1}}{\partial \beta} \sim p-p^{*}+O\left[\left(p-p^{*}\right)^{2}\right] .
$$

Hence, the first term in (C.6) may give rise to corrections of order $\left(p-p^{*}\right) L^{y_{1}-y_{2}}$. Thus, we also perform fits to

$$
\ln R^{\prime}=y_{2} \ln L+\sum_{n=0}^{n_{\max }} a_{n}\left(p-p^{*}\right)^{n} L^{n y_{1}}+L^{-y_{2}} \sum_{k=1}^{k_{\max }} b_{k}\left(p-p^{*}\right)^{k} L^{k y_{1}} .
$$

In Table 9 we report the results of the fits of $R_{\xi}^{\prime}$ and $U_{4}^{\prime}$ to (C.9) and (C.10). The inclusion of the analytic corrections significantly reduces the $\chi^{2}$ and changes slightly the estimates of $y_{2}$. Fits to (C.12) give results which are essentially equivalent to those obtained by fitting to (C.10). Comparing all results we obtain the estimate

$$
y_{2}=0.250(2)
$$

which is identical to that reported in [15].

Finally, we determine $\eta$. We compute it from the critical behavior of $\chi$ and, as in [15], from that of $Z \equiv \chi / \xi^{2}$. The results of the fits with and without analytic corrections are reported in Tables 10 and 11. The most stable results are obtained from fits of $\chi$ which take into account the analytic corrections. As final result we quote

$$
\eta=0.177(2)
$$

where the error is such to include the estimates of $\eta$ obtained from the analysis of $\ln Z$. This result is consistent with the estimate $\eta=0.180(5)$ reported in [15], but significantly more precise. 
Table 10 Estimates of $\eta$ at the MNP. We fix $y_{1}=0.66(1)$ and $p_{c}=0.89083(3)$. Results from fits of $\ln Z$ and $\ln \chi$ to (22) with $n_{\max }=2$ without analytic correction (in the case of $\ln Z$ the coefficient of $\ln L$ is of course $-\eta$ ). The error in parentheses is the sum of the statistical error and of the error due to uncertainty of $y_{1}$; the error in brackets gives the variation of the estimate as $p_{c}$ varies by one error bar

\begin{tabular}{rlllll}
\hline$L_{\min }$ & $\ln Z$ & & & \multicolumn{1}{l}{$\ln$} \\
\cline { 2 - 3 }$\chi^{2} / \mathrm{DOF}$ & $\eta$ & & $\chi^{2} / \mathrm{DOF}$ & $\eta$ \\
\hline 8 & $393 / 102$ & $0.1736(6)[12]$ & & $2653 / 102$ & $0.1752(2)[5]$ \\
12 & $272 / 96$ & $0.1747(7)[13]$ & & $2340 / 96$ & $0.1749(3)[5]$ \\
16 & $146 / 73$ & $0.1760(8)[14]$ & & $1342 / 73$ & $0.1751(3)[5]$ \\
24 & $68 / 50$ & $0.1776(10)[16]$ & & $761 / 50$ & $0.1752(4)[6]$ \\
32 & $30 / 27$ & $0.1782(12)[18]$ & & $110 / 27$ & $0.1761(4)[7]$ \\
\hline
\end{tabular}

Table 11 Estimates of $\eta$ at the MNP. We fix $y_{1}=0.66(1)$ and $p_{c}=0.89083(3)$. Results from fits of $\ln Z$ and $\ln \chi$ to (22) with $n_{\max }=2$ and $m_{\max }=1$. The error in parentheses is the sum of the statistical error and of the error due to uncertainty of $y_{1}$; the error in brackets gives the variation of the estimate as $p_{c}$ varies by one error bar

\begin{tabular}{|c|c|c|c|c|}
\hline \multirow[b]{2}{*}{$L_{\min }$} & \multicolumn{2}{|l|}{$\ln Z$} & \multicolumn{2}{|l|}{$\ln \chi$} \\
\hline & $\chi^{2} / \mathrm{DOF}$ & $\eta$ & $\chi^{2} / \mathrm{DOF}$ & $\eta$ \\
\hline 8 & $300 / 101$ & $0.1745(3)[11]$ & $83 / 101$ & $0.1767(1)[5]$ \\
\hline 12 & $117 / 95$ & $0.1763(3)[12]$ & 79/95 & $0.1768(1)[6]$ \\
\hline 16 & $60 / 72$ & $0.1776(4)[14]$ & $50 / 72$ & $0.1774(1)[6]$ \\
\hline 24 & $32 / 49$ & $0.1791(5)[15]$ & $36 / 49$ & $0.1771(1)[7]$ \\
\hline 32 & $15 / 26$ & $0.1794(9)[17]$ & $20 / 26$ & $0.1771(2)[7]$ \\
\hline
\end{tabular}

The results obtained here allow us to predict the behavior of the different transition lines close to the MNP. Standard scaling arguments predict that, close to the MNP, the transition lines are given by

$$
u_{1}\left|u_{2}\right|^{-\phi}=X_{ \pm},
$$

where $X_{+}$and $X_{-}$are two constants that refer to the lines which satisfy $T>T^{*}$ and $T<T^{*}$, respectively. They can be determined by considering the estimates of the critical points $p_{c}, T_{c}$ close to the MNP. The crossover exponent $\phi$ is equal to the ratio $y_{1} / y_{2}$. In the present case we have

$$
\phi=\frac{y_{1}}{y_{2}}=2.64(5) .
$$

We can use (C.15) to obtain an interpolation of our results up to $T=0$, which represents our best guess of the transition line, given the estimates of the critical points we have. For this purpose, we choose

$$
u_{2}(p, T)=\tanh (1 / T)-2 p+1
$$

so that $u_{2}=0$ along the $\mathrm{N}$ line, cf. (4). Thus, the critical line is given by the approximate expression

$$
p_{c}-p^{*}+a_{2}\left(T_{c}-T^{*}\right)^{2}=X_{-} u_{2}\left(p_{c}, T_{c}\right)^{\phi} .
$$

The left-hand side corresponds to the expansion of $u_{1}$, cf. (C.8), close to the MNP. Since $2<\phi<3$ the quadratic term proportional to $\left(T_{c}-T^{*}\right)^{2}$ is more relevant than the nonanalytic term which appears in the right-hand side of (C.18) and which therefore represents a 
next-to-leading contribution. Since $p_{c}-p^{*} \sim\left(T_{c}-T^{*}\right)^{2}$, the other quadratic terms appearing in the expansion of $u_{1}$ are subleading. The free parameters $a_{2}$ and $X_{-}$are fixed by requiring the line to go through the points $\left(p_{c}=0.8925(1), T_{c}=0.5\right)$ and $\left(p_{0}=0.897, T=0\right)$. We obtain $a_{2}=-0.0061$ and $X_{-}=0.0386$. The corresponding line is reported (dashed line) in Fig. 4. The interpolation (C.18) gives $p_{c}=0.89159$ at $\beta=1.55$, and the derivative $d p_{c} / d \beta=0.00180$ at $\beta=2$ which are in good agreement with the MC estimates $p_{c}=0.8915(2)$ at $\beta=1.55$, and $d p_{c} / d \beta=0.0020(3)$ at $\beta=2$ obtained in Sect. 3.

We have also determined an interpolation of the available numerical data [11,22] valid for $T>T^{*}$. A simple expression, which satisfies (C.15) and (3), is

$$
p_{c}=p^{*}+\left(\beta^{*}-\beta\right)^{2.64}\left(1.41484-4.25764 \beta+5.67965 \beta^{2}-2.77095 \beta^{3}\right),
$$

with $p^{*}=0.89083$ and $\beta^{*}=1.04962$. The corresponding line is reported (dashed line) in Fig. 4.

\section{References}

1. Edwards, S.F., Anderson, P.W.: Theory of spin glasses. J. Phys. F 5, 965-974 (1975)

2. Dennis, E., Kitaev, A., Landahl, A., Preskill, J.: Topological quantum memory. J. Math. Phys. 43, 44524505 (2002)

3. Kitaev, A.Yu.: Fault-tolerant quantum computation by anyons. Ann. Phys. (NY) 303, 2-30 (2003)

4. Nishimori, H.: Statistical Physics of Spin Glasses and Information Processing: An Introduction. Oxford University Press, London (2001)

5. Kawashima, N., Rieger, H.: Recent progress in spin glasses. In: Diep, H.T. (ed.) Frustrated Spin Systems. World Scientific, Singapore (2004). Chap. 6. arXiv:cond-mat/0312432

6. Hartmann, A.K.: Droplets in the two-dimensional $\pm J$ Ising spin glass. Phys. Rev. B 77, 144418 (2008)

7. Katzgraber, H.W., Lee, L.W., Campbell, I.A.: Effective critical behavior of the two-dimensional Ising spin glass with bimodal interactions. Phys. Rev. B 75, 014412 (2007)

8. Jörg, T., Lukic, J., Marinari, E., Martin, O.C.: Strong universality and algebraic scaling in twodimensional Ising spin glasses. Phys. Rev. Lett. 96, 237205 (2006)

9. Amoruso, C., Marinari, E., Martin, O.C., Pagnani, A.: Scalings of domain wall energies in two dimensional Ising spin glasses. Phys. Rev. Lett. 91, 087201 (2003)

10. Hartmann, A.K., Young, A.P.: Lower critical dimension of Ising spin glasses. Phys. Rev. B 64, 180404(R) (2001)

11. Hasenbusch, M., Parisen Toldin, F., Pelissetto, A., Vicari, E.: Universal dependence on disorder of twodimensional randomly diluted and random-bond $\pm J$ Ising models. Phys. Rev. E 78, 011110 (2008)

12. Blackman, J.A., Gonsalves, J.R., Poulter, J.: Properties of the two-dimensional random-bond $\pm J$ Ising spin glass. Phys. Rev. E 58, 1502-1507 (1998)

13. Nishimori, H.: Internal energy, specific heat and correlation function of the bond-random Ising model. Prog. Theor. Phys. 66, 1169-1181 (1981)

14. Ohzeki, M.: Precise locations of multicritical points for spin glasses on regular lattices. Phys. Rev. E 79, 021129 (2009)

15. Hasenbusch, M., Parisen Toldin, F., Pelissetto, A., Vicari, E.: Multicritical Nishimori point in the phase diagram of the $\pm J$ Ising model on a square lattice. Phys. Rev. E 77, 051115 (2008)

16. Nishimori, H.: Duality in finite-dimensional spin glasses. J. Stat. Phys. 126, 977-986 (2007)

17. Takeda, K., Sasamoto, T., Nishimori, H.: Exact location of the multicritical point in finite-dimensional spin glasses: a conjecture. J. Phys. A 38, 3751-3774 (2005)

18. Picco, M., Honecker, A., Pujol, P.: Strong disorder fixed points in the two-dimensional random-bond Ising model. J. Stat. Mech.: Theory Exp. P09006 (2006)

19. de Queiroz, S.L.A.: Multicritical point of Ising spin glasses on triangular and honeycomb lattices. Phys. Rev. B 73, 064410 (2006)

20. de Queiroz, S.L.A., Stinchcombe, R.B.: Correlation-function distributions at the Nishimori point of twodimensional Ising spin glasses. Phys. Rev. B 68, 144414 (2003)

21. Maillard, J.M., Nemoto, K., Nishimori, H.: Symmetry, complexity and multicritical point of the twodimensional spin glass. J. Phys. A 36, 9799-9825 (2003) 
22. Merz, F., Chalker, J.T.: Two-dimensional random-bond Ising model, free fermions, and the network model. Phys. Rev. B 65, 054425 (2002)

23. Nishimori, H., Falvo, C., Ozeki, Y.: Energy fluctuations at the multicritical point in two-dimensional spin glasses. J. Phys. A 35, 8171-8178 (2002)

24. Nishimori, H., Nemoto, K.: Duality and multicritical point of two-dimensional spin glasses. J. Phys. Soc. Jpn. 71, 1198-1199 (2002)

25. Honecker, A., Picco, M., Pujol, P.: Universality class of the Nishimori point in the $2 \mathrm{D} \pm J$ random-bond Ising model. Phys. Rev. Lett. 87, 047201 (2001)

26. Nobre, F.D.: Phase diagram of the two-dimensional $\pm J$ Ising spin glass. Phys. Rev. E 64, 046108 (2001)

27. Gruzberg, I.A., Read, N., Ludwig, A.W.W.: Random-bond Ising model in two dimensions: The Nishimori line and supersymmetry. Phys. Rev. B 63, 104422 (2001)

28. Arão Reis, F.D.A., de Queiroz, S.L.A., dos Santos, R.R.: Universality, frustration, and conformal invariance in two-dimensional random Ising magnets. Phys. Rev. B 60, 6740-6748 (1999)

29. Ozeki, Y., Ito, N.: Multicritical dynamics for the $\pm J$ Ising model. J. Phys. A 31, 5451-5465 (1998)

30. Migliorini, G., Berker, A.N.: Global random-field spin-glass phase diagrams in two and three dimensions. Phys. Rev. B 57, 426-431 (1998)

31. Cho, S., Fisher, M.P.A.: Criticality in the two-dimensional random-bond Ising model. Phys. Rev. B 55, 1025-1031 (1997)

32. Simkin, M.V.: Numerical study of competing spin-glass and ferromagnetic order. Phys. Rev. B 55, 11405-11408 (1997)

33. Singh, R.R.P., Adler, J.: High-temperature expansion study of the Nishimori multicritical point in two and four dimensions. Phys. Rev. B 54, 364-367 (1996)

34. Ozeki, Y., Nishimori, H.: Phase diagram of gauge glasses. J. Phys. A 26, 3399-3429 (1993)

35. Kitatani, H.: The verticality of the ferromagnetic-spin glass phase boundary of the $\pm J$ Ising model in the $p$ - $T$ plane. J. Phys. Soc. Jpn. 61, 4049-4055 (1992)

36. Le Doussal, P., Harris, A.B.: $\epsilon$ expansion for the Nishimori multicritical point of spin glasses. Phys. Rev. B 40, 9249-9252 (1989)

37. Le Doussal, P., Harris, A.B.: Location of the Ising spin-glass multicritical point on Nishimori's line. Phys. Rev. Lett. 61, 625-628 (1988)

38. Ozeki, Y., Nishimori, H.: Phase diagram of the $\pm J$ Ising model in two dimensions. J. Phys. Soc. Jpn. 56, 3265-3269 (1987)

39. Nishimori, H.: Geometry-induced phase transition in the $\pm J$ Ising model. J. Phys. Soc. Jpn. 55, 33053307 (1986)

40. Georges, A., Hansel, D., Le Doussal, P., Bouchaud, J.: Exact properties of spin-glasses. 1. 2D supersymmetry and Nishimori result. J. Phys. (Paris) 46, 1309 (1985)

41. Georges, A., Hansel, D., Le Doussal, P., Bouchaud, J.: Exact properties of spin-glasses. 2. Nishimori line-new results and physical implications. J. Phys. (Paris) 46, 1827-1836 (1985)

42. Georges, A., Hansel, D., Le Doussal, P., Maillard, J.M., Bouchaud, J.: Rigorous bounds for 2D disordered Ising models. J. Phys. (Paris) 47, 947-953 (1986)

43. McMillan, W.L.: Domain-wall renormalization-group study of the two-dimensional random Ising model. Phys. Rev. B 29, 4026-4029 (1984)

44. Amoruso, C., Hartmann, A.K.: Domain-wall energies and magnetization of the two-dimensional random-bond Ising model. Phys. Rev. B 70, 134425 (2004)

45. Wang, C., Harrington, J., Preskill, J.: Confinement-Higgs transition in a disordered gauge theory and the accuracy threshold for quantum memory. Ann. Phys. (NY) 303, 31-58 (2003)

46. Kawashima, N., Rieger, H.: Finite-size scaling analysis of exact ground states for $\pm J$ spin glass models in two dimensions. Europhys. Lett. 39, 85-90 (1997)

47. Domany, E.: Some results for the two-dimensional Ising model with competing interactions. J. Phys. C: Solid State 12, L119-L123 (1979)

48. Nishimori, H.: Derivatives and inequalities for order parameters in the Ising spin glass. J. Phys. A 35, 9541-9548 (2002)

49. Shalaev, B.N.: Critical behavior of the 2-dimensional Ising model with random bonds. Phys. Rep. 237, 129-188 (1994)

50. Shankar, R.: Exact critical behavior of a random bond two-dimensional Ising model. Phys. Rev. Lett. 58, 2466-2469 (1987). Erratum 59, 380 (1987)

51. Ludwig, A.W.W.: Comment on "Exact critical behavior of a random-bond two-dimensional Ising model”. Phys. Rev. Lett. 61, 2388 (1988). Erratum 62, 980 (1987)

52. Ceccatto, H.A., Naon, C.: Comment on "Exact critical behavior of a random-bond two-dimensional Ising model". Phys. Rev. Lett. 61, 2389 (1988)

53. Ludwig, A.W.W., Cardy, J.L.: Perturbative evaluation of the conformal anomaly at new critical points with applications to random systems. Nucl. Phys. B 285, 687-718 (1987) 
54. Cardy, J.L.: Logarithmic corrections to finite-size scaling in strips. J. Phys. A 19, L1093-L1098 (1986). Erratum 20, 5039 (1987)

55. Geyer, C.J.: Markov chain Monte Carlo maximum likelihood. In: Keramidas, E.M. (ed.) Computer Science and Statistics: Proc. of the 23rd Symposium on the Interface, p. 156. Interface Foundation, Fairfax Station (1991)

56. Hukushima, K., Nemoto, K.: Exchange Monte Carlo method and application to spin glass simulations. J. Phys. Soc. Jpn. 65, 1604-1608 (1996)

57. Earl, D.J., Deem, M.W.: Parallel tempering: theory, applications, and new perspectives. Phys. Chem. Chem. Phys. 7, 3910-3916 (2005)

58. Hasenbusch, M., Pelissetto, A., Vicari, E.: The critical behavior of three-dimensional Ising spin glass models. Phys. Rev. B 78, 214205 (2008)

59. Salas, J., Sokal, A.D.: Universal amplitude ratios in the critical two-dimensional Ising model on a torus. J. Stat. Phys. 98, 551-588 (2000)

60. Hasenbusch, M., Parisen Toldin, F., Pelissetto, A., Vicari, E.: The universality class of 3D site-diluted and bond-diluted Ising systems. J. Stat. Mech.: Theory Exp. P02016 (2007) 